\title{
Complex Band Structure and Tunneling through Ferromagnet/Insulator/Ferromagnet Junctions
}

\author{
Ph. Mavropoulos, N. Papanikolaou, and P. H. Dederichs \\ Institut für Festkörperforschung, Forschungszentrum Jülich, D-52425 Jülich, Germany
}

(Received 24 January 2000)

\begin{abstract}
We investigate the importance of metal-induced gap states for the tunneling of metal electrons through epitaxial insulator films. By introducing an imaginary part $\kappa$ to the wave vector in order to describe the decay of the wave function in the insulator, we obtain the complex band structure in the gap region. The spectrum of the decay parameters $\kappa$ is calculated for the semiconductors $\mathrm{Si}, \mathrm{Ge}, \mathrm{GaAs}$, and $\mathrm{ZnSe}$. In most cases, for large enough film thicknesses the tunneling is dominated by states of normal incidence on the interface. Possible exceptions are considered. Based on our conclusions, we discuss the spindependent tunneling in $\mathrm{Fe} /$ semiconductor/Fe (001) junctions.
\end{abstract}

PACS numbers: $73.40 . \mathrm{Gk}, 73.40 . \mathrm{Rw}$

Tunneling magnetoresistance (TMR) is one of the fields where basic physics research and technological applications actually meet. Julliere [1] first showed that in ferromagnet/insulator/ferromagnet (FM/I/FM) junctions the transmission is spin dependent and thus the conductance depends on the parallel or antiparallel orientation of the magnetization of the two FM leads. More recently, Miyazaki and Tezuka [2] and Moodera et al. [3] were able to obtain in room-temperature experiments a TMR ratio as high as $20 \%$. The effect of tunneling magnetoresistance was thus rediscovered, and there is great interest for potential applications, especially as nonvolatile magnetic random access memories.

While the effect of giant magnetoresistance in magnetic multilayers has been extensively studied, the research on the TMR effect in FM/I/FM junctions is still in an early stage. In particular, theoretical calculations based on models [4-6] have shed some light on various aspects of the effect, but only recently first-principles calculations of the electronic structure [7] and electronic transmission [8] have been reported. From the analysis of the $\mathrm{Fe} / \mathrm{ZnSe} / \mathrm{Fe}$ system in the latter work it becomes clear that a simple barrier model is insufficient for a correct description of the spin-dependent tunneling in such epitaxial junctions.

In this paper we demonstrate that the tunneling through insulators can be understood in terms of the metal-induced gap states (MIGS) introduced by Heine [9] and that the framework to investigate them is the complex band structure of the insulator in the gap region. To our knowledge the connection of the complex band structure with tunneling through junctions and the resulting predictions have not been pointed out previously. As model systems for our discussion we will use $\mathrm{Fe} / \mathrm{I} / \mathrm{Fe}$ (001) sandwiches, where "I" refers to the semiconductors $\mathrm{Si}, \mathrm{Ge}, \mathrm{GaAs}$, and $\mathrm{ZnSe}$. Note that our calculations are restricted to crystalline barriers and do not allow applications to amorphous barriers such as $\mathrm{Al}_{2} \mathrm{O}_{3}$ which are mostly used in experiments.

In the bulk of a crystal, the periodicity demands that the Bloch $\mathbf{k}$ vectors are purely real. But near a crystal surface or interface one can match a wave function of complex $\mathbf{k}$ with one outside the crystal, and thus surface or interface evanescent states or MIGS are described [9]. The energy bands corresponding to complex k's are called complex bands. In particular, insulator surfaces can host such states with their energy in the band gap. Although they can exist only at a surface or interface, their general properties can be derived from the bulk band structure of the material, if one formally allows complex $\mathbf{k}$ vectors to characterize the solutions of the Schrödinger equation for the periodic bulk potential. The reason is that the interface-induced changes in the charge density and in the potential are confined in their greatest part to the first one or two monolayers, but the evanescent wave functions themselves extend over many layers into the crystal, and therefore represent solutions of the bulk Schrödinger equation.

One can split the wave vector into a part parallel to the interface of interest, $\mathbf{k}_{\|}$, which is conserved during scattering, and a part perpendicular to the interface, $k_{z}$. Here, perfect epitaxial growth is assumed, so that the twodimensional periodicity is valid for both half spaces. Then, for each real $\mathbf{k}_{\|}$, one can find the dispersion relations $E=E\left(k_{z}\right)$, allowing complex $k_{z}=q+i \kappa$. We denote the imaginary part $\kappa$ as the decay parameter; the corresponding wave functions will fall off as $\sim e^{-\kappa z}$. The solutions of the Schrödinger equation for a certain $\mathbf{k}_{\|}$and arbitrary complex $k_{z}$, which are associated with a realvalued energy form the so-called real lines in the threedimensional $(q, \kappa, E)$ space.

The analytic properties of real lines were studied by Kohn [10] and Heine [9], and the topology of the complex bands was studied by Chang [11]. The solution of the Schrödinger equation always gives them in pairs of $\pm \kappa$, but the physically relevant ones are only those that decay into the crystal bulk. Real lines depart from the real $q$ axis wherever there is a local maximum or minimum of the real band structure with respect to $q$, and they can either extend in energy to $-\infty$, forming free-electronlike 
parabolic solutions, or connect back to other extrema of the real band structure, forming closed loops.

In the case of a metal-insulator (or metal-semiconductor) interface, the Fermi level, $E_{F}$, lies in the insulator band gap. For a metal/insulator/metal junction, metallic states at $E_{F}$, which are responsible for the transport, couple to the insulator states of complex $k_{z}$. Clearly, the MIGS at $E_{F}$ will play the central role in tunneling. Of these, only those are effective that have a reasonably small decay parameter. In fact, in the limit of large barrier thickness only the state with the smallest decay parameter, $\kappa_{\min }$, survives and contributes to the tunneling current. The determination of $\kappa_{\min }$ can proceed in two steps. First, for given values of $\mathbf{k}_{\|}$and $E_{F}$, one has to determine, out of the infinitely many solutions with different decay parameters, the one with the smallest $\kappa>0$ value. In the second step, one can then vary $\mathbf{k}_{\|}$and search for the absolute minimum $\kappa_{\min }$ compatible with $E_{F}$. Of course, states with $\kappa$ close enough to $\kappa_{\min }$ will also be important. Of prime importance is then the character of these states, e.g., their symmetry and $\mathbf{k}_{\|}$ values. This information is decisive in determining which metallic states of the lead, whether bulk or interface states, can couple to them and thus be important for tunneling. In the case of a FM/I/FM junction the majority-spin bands of the leads can have different symmetry properties at the Fermi level than the minority-spin ones, and thus couple differently to the MIGS of $\kappa_{\min }$; this leads to tunneling magnetoresistance.

The method used in this paper for the calculation of the complex band structure is the simplest possible: We apply an empirical local pseudopotential plane-wave technique, with the form factors taken from Ref. [12]. Since the form factors have been fitted to spectroscopic data, this method gives a reasonably good description of the band structure in the gap region (this being the most important for the tunneling). Clearly, local density functional calculations give a much better overall description of the band structure, but they have the well-known deficiency of underestimating the band gap by about $50 \%$. Thus the above method is well suited for our purpose.

We may begin our discussion with the examination of the complex band structure of $\mathrm{ZnSe}(001)$ films in the $k_{z}$ direction for $\mathbf{k}_{\|}=0$, shown in Fig. 1. The nonreal bands have been drawn for $q=0$ and $q=2 \pi / a$ ( $a$ being the lattice constant), omitting for the sake of clarity in the figure other complex bands that would depart from local maxima or minima in between. From the aspect of symmetry, the nonreal bands inherit the symmetry properties of the real bands they are connected to. Also, the curvature at the connecting points must be the same for the real band and its nonreal extension, due to analyticity.

We now choose an energy in the gap. Then, at least for the complex bands drawn in the picture, the one with the smallest $\kappa$ will be that of symmetry $\Delta_{1}$ (the identity representation) connecting the top of the $\Delta_{1}$ valence band to the bottom of the $\Delta_{1}$ conduction band. We expect this to

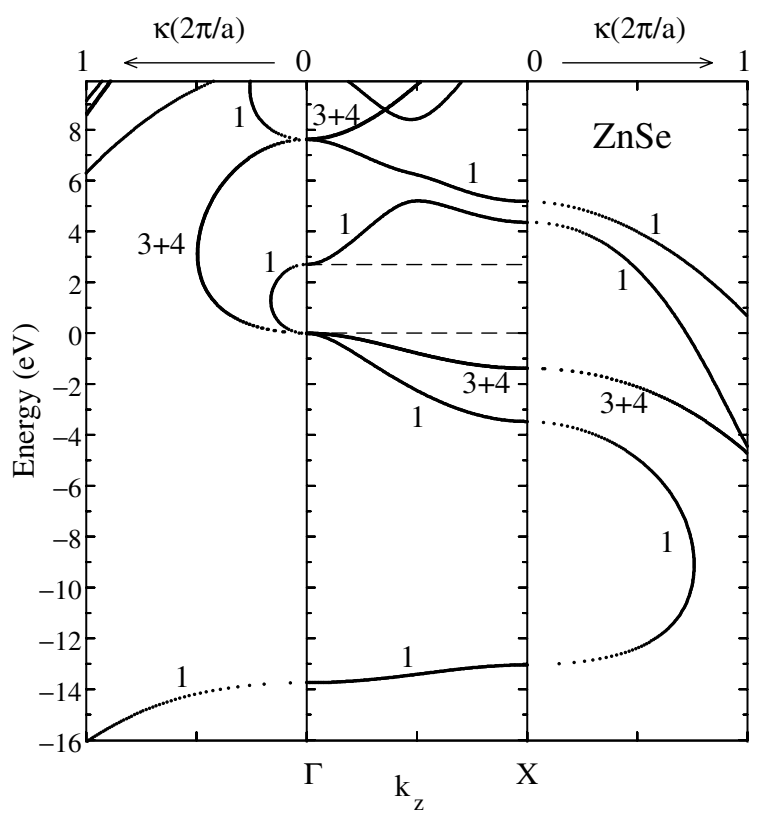

FIG. 1. The complex band structure of $\mathrm{ZnSe}$, for $\mathbf{k}_{\|}=0$, at $q=0$ (left panel) and $q=2 \pi / a$ (right panel). The real lines are forming loops and free-electronlike parabolas (see also text).

be true quite generally when we are dealing with a direct gap characterized by a $\Delta_{1}$ valence band maximum and a $\Delta_{1}$ conduction band minimum. The loop connecting these particular extrema will then be smaller than any other one, and so will be the corresponding value of the decay parameter. The inverted parabola beginning from the lowest conduction band at $q=2 \pi / a$ starts both higher in energy and with small curvature, and shoots off to very high $\kappa$ values. Additionally, infinitely many other inverse-parabolic states exist starting from higher bands, having much larger $\kappa$ values. Departing from the $\bar{\Gamma}$ point, we know that the valence states are lowered energetically, the conduction states are raised, and the gap increases. For this reason it is expected that the loops at $q=0$ become larger for nonzero $\mathbf{k}_{\|}$values, and the corresponding values of $\kappa$ increase. Note that a simple barrier model for tunneling would greatly overestimate the decay parameter, because it would correspond to an inverted parabolic solution and thus would not predict the increased bending of the $\Delta_{1}$ loop that results in very small $\kappa$ values.

By proceeding with the search for $\kappa_{\min }$, one can scan the whole two-dimensional surface Brillouin zone at a given energy. One would thus form a constant-energy surface in the $\left(\mathbf{k}_{\|}, \kappa\right)$ space. This consists of many branches, corresponding to the (infinitely) many different complex bands for any given $\mathbf{k}_{\|}$, but our attention should be focused on the lowest-lying one. This is shown in Fig. 2 for four materials, namely, $\mathrm{Si}, \mathrm{Ge}, \mathrm{GaAs}$, and $\mathrm{ZnSe}$, for a typical value of $E_{F}$ in the middle of the gap. We see that in all four cases the decay parameter is minimized at the $\bar{\Gamma}$ point. We also see that the difference of $\kappa$ at the flatter part of the branch and at $\mathbf{k}_{\|}=0\left(\kappa_{\min }\right)$ increases 

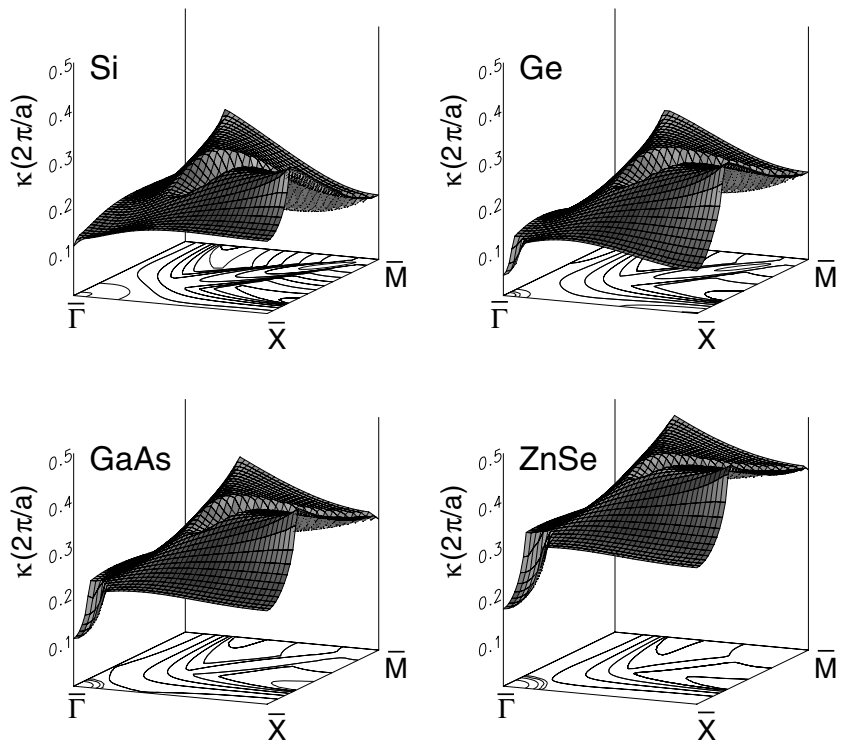

FIG. 2. The constant-energy surfaces $\kappa=\kappa\left(\mathbf{k}_{\|}\right)$drawn in one-fourth of the surface Brillouin zone for $\mathrm{Si}, \mathrm{Ge}, \mathrm{GaAs}$, and ZnSe. Only the lowest positive branches are drawn, for an energy in the middle of the band gap. The decay parameter has its minimum at the $\bar{\Gamma}$ point in all four cases.

monotonically with the band gap, this also following the order $\mathrm{Si}<\mathrm{Ge}<\mathrm{GaAs}<\mathrm{ZnSe}$.

Although it is evident that in the presented cases the state $\mathbf{k}_{\|}=0$ will penetrate into the insulator farther than any other, we can see in Fig. 2 that the states belonging to the flatter part of the branches outnumber by far the ones near $\mathbf{k}_{\|}=0$. This becomes clearer if we introduce the $\kappa$-resolved density of states $n(\kappa)$, with $n(\kappa) d \kappa$ the number of states with $\kappa$ values in the infinitesimal interval $(\kappa, \kappa+d \kappa)$, for a given energy. This is shown in Fig. 3 for $\mathrm{ZnSe}$, for the energy in the middle of the gap. In this plot, all branches of the constant-energy surface have been taken into account. We see that for $\kappa>0.3(2 \pi / a)$ the flat parts of the branches give a large contribution to $n(\kappa)$. In order to obtain a feeling about the relative importance of states with different $\kappa$ values for a given insulating film thickness $d$, we have plotted in Fig. 4 the quantity $n(\kappa) e^{-2 \kappa d} /\left[n\left(\kappa_{\min }\right) e^{-2 \kappa_{\min } d}\right]$, since the current will be attenuated by $e^{-2 \kappa d}$. We see that the high- $\kappa$ states with $\kappa>\pi / a$ are already damped out for eight monolayers of ZnSe $(d=2 a)$. In this region of intermediate thickness the current is still dominated by the flat branches in Figs. 2 and 3 with contributions arising from the whole surface Brillouin zone. For larger thicknesses, i.e., 16 or 24 monolayers, the distribution contracts close to the $\kappa_{\min }$ value. This represents the asymptotic limit, where the current is practically carried only by the $\Delta_{1}$ MIGS around the $\bar{\Gamma}$ point and shows the slowest possible decrease $\sim e^{-2 \kappa_{\min } d}$. Thus for large thicknesses the decrease is considerably slower than in the intermediate region of 5-10 monolayers.

Now, the following question arises: Is it conceivable that in some cases $\kappa_{\min }$ is reached at some $\mathbf{k}_{\|} \neq 0$ ? The

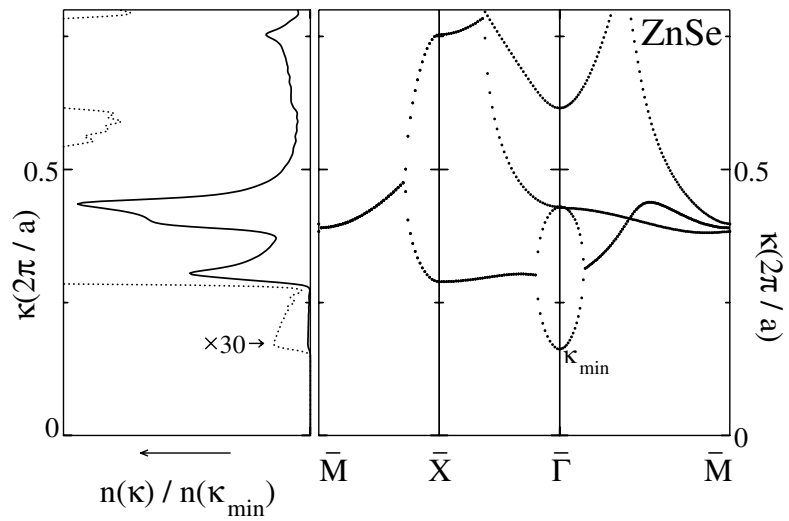

FIG. 3. The $\kappa$-resolved density of states $n(\kappa)$ for ZnSe (left), for a choice of $E_{F}$ in the middle of the band gap, together with the constant-energy branches in high-symmetry directions of the surface Brillouin zone (right).

answer is, yes. Take a semiconductor with an indirect absolute gap, such as $\mathrm{Si}$ or Ge. In these two, the top of the valence band is still at $\Gamma$, but the bottom of the conduction band is in the $\Gamma-X$ direction near $X$, for $\mathrm{Si}$, and at the edge of the Brillouin zone at $L$, for Ge. For the corresponding $\mathbf{k}_{\|}$'s, $E(q)$ has of course a minimum at these points, and so complex bands depart from there. We can choose an energy in the gap arbitarily close to this minimum, and thus make $\kappa$ arbitarily small at the specific $\mathbf{k}_{\|}$. Of course, as we decrease the energy we will eventually reach a value close enough to the top of the valence band where $\kappa_{\min }=\kappa\left(\mathbf{k}_{\|}=0\right)$ again. Therefore in these cases the position of the Fermi level decides which $\mathbf{k}_{\|}$value has the smallest $\kappa$. In the case of Ge, the complex bands at $\Gamma$ with $\mathbf{k}_{\|}=0$ dominate practically in the whole band gap region, while for $\mathrm{Si}$ the transition from $\Gamma$ to $\mathrm{X}$ occurs at $0.35 \mathrm{eV}$ below the conduction band minimum.

The results obtained here for $\mathrm{Ge}, \mathrm{GaAs}$, and $\mathrm{ZnSe}$ (that the minimum decay constant is determined by the $\Delta_{1}$ loop for $\mathbf{k}_{\|}=0$ ) should be valid for a large class of semiconductors. In short the tendency for a direct gap increases

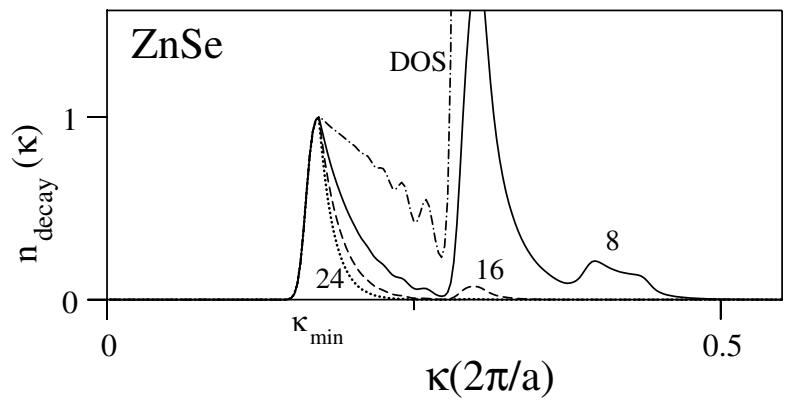

FIG. 4. The quantity $n_{\text {decay }}(\kappa)=\left[n(\kappa) / n\left(\kappa_{\min }\right)\right] e^{-2\left(\kappa-\kappa_{\min }\right) d}$, a measure of the relative importance of the states of various $\kappa$ to tunneling, for several thicknesses $d$ of the $\mathrm{ZnSe}$ film (8, 16, and 24 monolayers). For comparison, just the normalized $n(\kappa) / n\left(\kappa_{\min }\right)$ is shown (dashed-dotted line labeled DOS). $E_{F}$ is taken in the middle of the band gap. 
with increasing nuclear charge and/or with increasing ionicity of the compound, and in these cases the $\Delta_{1}$ loop at the $\Gamma$ point should determine the tunneling. In addition to the above semiconductors, another important example showing the same behavior is $\mathrm{MgO}$, which, similar to $\mathrm{GaAs}$ and $\mathrm{ZnSe}$, grows epitaxially on $\mathrm{Fe}(001)$. The band structure of $\mathrm{MgO}$ has the same topology as the one of GaAs and $\mathrm{ZnSe}$, and our calculations show that also for $\mathrm{MgO}$ the tunneling is determined by the $\Delta_{1}$ loop at the $\Gamma$ point. However, as we have seen for $\mathrm{Si}$, there are important exceptions, in particular when light elements are involved. Then other $\mathbf{k}_{\|}$ points could be decisive.

On the basis of the conclusions thus far, we will now describe the situation in a particular ideal tunneling experiment. Consider an ideal junction made of a $\mathrm{Fe} / \mathrm{ZnSe} / \mathrm{Fe}$ (001) sandwich, with perfect two-dimensional translational symmetry parallel to the $x y$ surface and temperatures low enough that we must handle only the ground-state properties. An infinitesimal bias on the junction would produce an infinitesimal tunneling current affecting only the electrons of the Fermi level. The states relevant for tunneling are those near the $\bar{\Gamma}$ point.

Looking at the complex band structure of $\mathrm{ZnSe}$ for $\mathbf{k}_{\|}=$ 0 (Fig. 1), we see that the smallest loop, corresponding to $\kappa_{\min }$, is characterized by a $\Delta_{1}$ symmetry. If any electron originating from the Fe lead is to tunnel via this state, it must belong to a state in Fe that is also characterized by $\Delta_{1}$ symmetry, so that it can couple to this one. Note here that we refer to the symmetry group of the combined system, which in our case is $C_{2 v}$, with the rotations taking place in the $x y$ plane. In the Fe lead, the $k$-space direction $\Delta$ corresponding to the (001) surface is $\Gamma-H$. Looking at the band structure of $\mathrm{Fe}$ in this direction (see, e.g., Ref. [13]), we see that there are relevant states of symmetry $\Delta_{1}$ at $E_{F}$ only among the majority-spin bands (there are also states of the $C_{4 v}$ symmetry $\Delta_{2}^{\prime}$, which is compatible to the $C_{2 v} \Delta_{1}$, but they are $d_{x y}$-like and are expected to couple very poorly to the semiconductor gap states). These would then couple to the $\Delta_{1}$ complex state in $\mathrm{ZnSe}$ and pass to the other side. Electrons of other symmetries would be cut off by a thick enough film. But, when the electron has reached the interface of the second lead, it will or will not be allowed to continue, depending on the relative magnetic orientation of the two leads. If their orientation is parallel, it will couple onto the $\Delta_{1}$ state and continue. But, in the case of antiparallel alignment, the former majority band will now be the minority one; and there is no state in the minority band at $E_{F}$ to couple to the $\Delta_{1}$ complex state from which the electron emerges. Then there will be no conductance. These arguments are exact for the state of $\kappa_{\min }$, at $\mathbf{k}_{\|}=0$, but for reasons of continuity they will almost hold for $\mathbf{k}_{\|}$ near the $\bar{\Gamma}$ point, where the decay parameter is very close to $\kappa_{\min }$.

Our analysis is fully consistent with the ab initio results of MacLaren et al. [8] which in fact motivated this study. There, in the case of parallel alignment of the leads, the transmission is found to be concentrated to the states around and at $\mathbf{k}_{\|}=0$ for the majority-spin channel and, in fact, more and more as the film thickness is increased. Furthermore, the (low) transmission of the minority-spin electrons is confined to a ring around $\mathbf{k}_{\|}=0$. This corresponds to states that do have a low enough value of $\kappa$, close to $\kappa_{\min }$, but also have at least some nonzero projection to symmetries other than $\Delta_{1}$, so that they can couple to the minority-spin states of leads. In the case of antiparallel alignment, again, the (low) conductance is confined to states forming a ring around $\mathbf{k}_{\|}=0$, and the same argument as before applies.

In summary, we have shown that conclusions about the tunneling in FM/I/FM epitaxial systems can be drawn by a simple inspection of the complex band structure of the insulator in the gap region. For the above cases this has been obtained by using simple pseudopotential techniques, and as a matter of fact it can be understood, in a first approximation, just by looking at the real band structure and by noticing the symmetry of the real bands and their curvature at the minima and maxima. Thus it constitutes a very useful tool for the understanding and prediction of tunneling phenomena.

[1] M. Julliere, Phys. Lett. 54A, 225 (1975).

[2] T. Miyazaki and N. Tezuka, J. Magn. Magn. Mater. 139, L231 (1995).

[3] J. S. Moodera, L. R. Kinder, T. M. Wong, and R. Meservey, Phys. Rev. Lett. 74, 3273 (1995).

[4] J. C. Slonczewski, Phys. Rev. B 39, 6995 (1989).

[5] J. M. MacLaren, X.-G. Zhang, and W. H. Butler, Phys. Rev. B 56, 11827 (1997).

[6] S. Zhang and P. M. Levy, Phys. Rev. Lett. 81, 5660 (1998).

[7] W. H. Butler, X.-G. Zhang, X. Wang, J. van Ek, and J. M. MacLaren, J. Appl. Phys. 81, 5518 (1997).

[8] J. M. MacLaren, X.-G. Zhang, W. H. Butler, and Xindong Wang, Phys. Rev. B 59, 5470 (1999).

[9] V. Heine, Proc. Phys. Soc. London 81, 300 (1962); Surf. Sci. 2, 1 (1964); Phys. Rev. 138, A1689 (1965).

[10] W. Kohn, Phys. Rev. 115, 809 (1959).

[11] Y.-C. Chang, Phys. Rev. B 25, 605 (1982).

[12] Marvin L. Cohen and T. K. Bergstresser, Phys. Rev. 141, 789 (1966).

[13] J. Callaway and C. S. Wang, Phys. Rev. B 16, 2095 (1977). 